\title{
Implementing evidence-based interventions to prevent readmissions in the real world
}

\author{
Nate L. Ewigman, $P h D, M P H^{1,2}$, Lea Vella, $P h D, M P H^{7}$, and Jessica A. Eng, MD, MS ${ }^{1,3}$ \\ 'San Francisco VA Healthcare System, San Francisco, CA, USA; ${ }^{2}$ Department of Psychiatry, University of California San Francisco, San Francisco, CA, \\ USA; ${ }^{3}$ Division of Geriatrics, University of California San Francisco, San Francisco, CA, USA.
}

$\mathrm{J}$ Gen Intern Med 33(5):578-80

DOI: $10.1007 / \mathrm{s} 11606-018-4351-8$

(c) Society of General Internal Medicine (outside the USA) 2018

$\mathrm{S}$ uboptimal transitions from the hospital to the community often cause hospital readmissions. Developing specialized teams and standardized processes during transitions are increasingly seen as potential approaches to reducing acute care utilization and improving clinical outcomes. ${ }^{1}$ Some interventions have shown promising results in randomized controlled trials, although systematic reviews have reported mixed results. $^{2}$ While the literature on implementing such interventions is nascent, there are a few principles to which successful programs adhere.

Identification based on triangulation of data. The two common methods of identifying patients for intervention are based either on (1) clinician judgment or (2) administrative data. These two methods often result in substantially different cohorts. For example, administrative data usually cannot take into consideration social support and health literacy, both of which are key to patients successfully following discharge instructions including medication regimens and attending follow up appointments. While administrative data can accurately predict acute care utilization, it cannot capture the critical, yet elusive, concept of modifiability. Clinical providers have knowledge of a patient's readiness to change and insight into their psychosocial context. They tend to identify patients for intervention who are older and have problems with mental health, substance use, medical decision-making, and care coordination. However, provider-led identification tends to only modestly predict those at risk of rehospitalization. ${ }^{3}$ Ideally, patients are identified through use of a triangulated approach in which clinical and administrative approaches are melded to account for the inherent weaknesses in each method. ${ }^{1}$

Intervention intensity based on risk stratification. Once identified, patients should be stratified by risk to allow for right-sizing of intervention. While home visits can be an effective tool to address medication adherence and low health literacy issues that often play a role in poor transitions, they are

Published online February 27, 2018 time-intensive and expensive. Telephone-based interventions are less time-intensive and less costly but might not completely assess and address the needs of complex patients. Stratifying based on potential needs can guide a stepped-care, population-based interventional approach ${ }^{1}$ that provides the optimal intervention dose for the most benefit.

Use of evidence-based processes. There are many evidencebased models to use or draw from although the literature is limited about these models' implementation outside research trials. 2, 4, 5 Thoughtful consideration of the type of outcomes most important to the patient and healthcare system can guide the choice of specific evidence-based interventions. Certain healthcare systems might want to focus on particular performance areas, such as congestive heart failure readmissions, while others might want to broaden the focus to supporting primary care in quality of care for older adults, and the choice of model and specific intervention processes should be aligned with the expected outcomes.

Adaptation of intervention to healthcare system. Intervention feasibility will be affected by many factors. Without adaptation, interventions usually come to settings with a poor fit, resisted by individuals who will be affected, and requiring an active process to engage individuals in order to accomplish implementation. ${ }^{6,7}$ The size of a hospital's catchment area will determine the feasibility of home visits. Relationship strength with referring primary care practices will determine their interest in interacting with teams designed to ease the transition from inpatient back to these practices. In addition, when considering implementation of telephone-based or home-based interventions, hospitals should consider the current resources invested in these areas, such as disease-specific telehealth programs and home-based primary care, and how the new intervention will enhance rather than duplicate current services.

Use of quadruple aim. While different stakeholders may want to focus on particular outcomes (e.g., cost), intervention goals should consider the Quadruple Aim: costs, patient satisfaction and experience, quality and outcomes, and provider burnout. $^{8,9}$ While having such variety of goals makes evaluation of outcomes difficult, in our experience, this 
multi-factorial approach is both the logical and ethical approach towards delivering effective care.

In this issue of JGIM, Hoyer et al. present a prospective observational study that represents their health system's commitment to reducing readmissions at two Maryland hospitals through risk-stratifying all patients hospitalized for a nearly three-year period. This study signifies a major commitment to reducing risk of readmission among all patients. We believe this study is an example of an intervention that meets several of the principles we have articulated.

Their selection strategy triangulates multidisciplinary team assessment and the evidence-based Early Screen for Discharge Planning (ESDP) score. A greater percentage of patients with high ESDP scores were referred to the more intensive intervention (22\% of those referred to the lower intensity Patient Access Line [PAL] intervention vs. 35\% of those referred to the higher intensity Transition Guide [TG] intervention), suggesting that the clinical and team assessment had an impact on risk stratification decisionmaking. Consistent with literature showing additive predictive value of more data points, ${ }^{9}$ it is likely that the clinical and administrative data inform one another to appropriately risk stratify. They then stratified patients into low- and high-risk categories, based on the team or ESDP assessment. This allowed for a stepped care approach to right-size a lower and higher intensity intervention: those considered higher need were offered a nurse $\mathrm{TG}$, and those who were low-risk were assigned the PAL intervention, a post-discharge phone call from a trained nurse. Additionally, the intervention design also incorporated a population-based strategy by providing intervention for all patients. This approach recognizes the universal vulnerability experienced by patients being discharged from the hospital. ${ }^{10}$ Lastly, this intervention was designed to improve cost through reducing readmissions but also aimed to improve the experiences and health outcomes of patients at a vulnerable point in their lives. In our experience, recurrent readmissions can be a source of perceived failure and ineffectiveness, hopelessness, and burnout for providers and staff across the healthcare system, making this intervention important for the providers of care as well as making it consistent with the quadruple aim.

Hoyer et al. ${ }^{11}$ showed that both the higher and lower intensity interventions reduced readmissions. Compared to those who received each intervention, those who did not receive them had greater odds of being readmitted ( 1.83 for TG, 1.27 for PAL). These results are impressive given the general difficulty reducing overall 30-day readmissions, rather than focusing disease-specific cohorts. However, the results are tempered by the methodological concerns common to many studies looking at studying interventions in real world circumstances without the benefit of randomization. The use of a comparison group is preferable to not having one; however, the choice of individuals who did not receive the intervention (presumably due to refusal or inability to contact) as the comparison makes it likely that intervention patients were the "lower hanging fruit," i.e., those more likely to benefit, particularly in the TG group. Another methodological concern involves the mixture of low- and high-risk individuals in the PAL arm. Some high-risk participants were first referred to the TG group but declined, and were then offered the PAL intervention. This contamination between groups makes it difficult to describe the PAL group as a low-risk cohort but also makes it more impressive that the PAL intervention significantly reduced readmissions compared to those who did not receive it. Lastly, the authors present data suggesting that those who did not receive interventions were the "hardest to reach" and at highest risk for readmission. Adjusting for sociodemographic characteristics would have been helpful in determining the effectiveness of the intervention.

Overall, we congratulate Hoyer et al. on their work and institutional commitment to reducing unnecessary and costly readmissions. This intervention demonstrates the use of triangulated identification of complex patients who were riskstratified to evidence-based interventions aimed at improving the health and experience of patients. It reduced cost to the health system and burden on providers. While there are likely not universal interventions that can be applied regardless of local and regional system and patient variation, Hoyer et al. have demonstrated the significant impact of high quality evidence-based interventions that are thoughtfully adapted to the local context.

Corresponding Author: Jessica A. Eng, MD, MS; San Francisco VA Healthcare System, San Francisco, CA, USA (e-mail: jessica.eng@va. gov).

\section{Compliance with Ethical Standards:}

Conflict of Interest: The authors have no conflicts of interest to declare.

\section{REFERENCES}

1. Hong CS, Abrams MK, Ferris TG. Toward increased adoption of complex care management. N Engl J Med. 2014;371:491-493.

2. Hansen LO, Young RS, Hinami K, Leung A, Williams MV. Interventions to reduce 30-day rehospitalization: a systematic review. Ann Intern Med. 2011;155:520-28.

3. Allaudeen N, Schnipper JL, Orav EJ, Watchter RM, Vidyarthi AR. Inability of providers to predict unplanned readmissions. J Gen Intern Med. 2011;26:771-776.

4. Coleman EA, Parry C, Chalmers S, Min SJ. The care transitions intervention: results of a randomized controlled trial. Arch Intern Med. 2006;166:1822-1828.

5. Snow V, Beck D, Budnitz T, Miller DC, Potter J, Wears RL, et al. Transitions of Care Consensus Policy Statement from the American College of Physicians, Society of General Internal Medicine, Society of Hospital Medicine, American Geriatrics Society, American College of Emergency Physicians, Society of Academic Emergency Medicine. J Gen Intern Med. 2009;24:971-976.

6. Damschroder LJ, Aron DC, Keith RE, Kirsh SR, Alexander JA, Lowery JC. Fostering implementation of health services research findings into practice: a consolidated framework for advancing implementation science. Implement Sci 2009;4:50-65. 
7. Ritchie C, Andersen R, Eng J, Garrigues SK, Intinarelli G, Kao $\mathbf{H}$, et al. Implementation of a team-based complex care support health care model at an academic medical center: impact on health care utilization and quality of life. PLoS One 2016; 11(2):e0148096.

8. Bodenheimer T, Sinsky C. From triple to quadruple aim: care of the patient requires care of the provider. Ann Fam Med. 2014;12:573-576.

9. Billings J, Georghiou T, Blunt I, Bardsley M. Choosing a model to predict hospital admission: an observational study of new variants of predictive models for case finding. BMJ Open 2013;3 e003352.
10. Krumholz HM. Post-hospital syndrome-an acquired, transient condition of generalized risk. New Engl J Med 2013;368:100-102.

11. Hoyer EH, Brotman, DJ, Apfel A, Leung C, Boonyasai RT, Richardson M, Lepley D, Deutschendorf A. Improving outcomes after hospitalization: a prospective observational multicenter evaluation of care coordination strategies for reducing 30-day readmissions to Maryland hospitals. J. Gen Intern Med. 2017 doi:https://doi.org/10.1007/s11606-017-4218-4 